\title{
A single-mask thermal displacement sensor in MEMS
}

R.P. Hogervorst ${ }^{1,2}$, B. Krijnen ${ }^{1}$, D.M. Brouwer ${ }^{1,3}$, J.B.C. Engelen ${ }^{4}$, U. Staufer ${ }^{2}$

${ }^{1}$ DEMCON Advanced Mechatronics, Oldenzaal, the Netherlands

${ }^{2}$ Precision and Microsystems Engineering, TU Delft, the Netherlands

${ }^{3}$ Mechanical Automation, IMPACT, University of Twente, the Netherlands

${ }^{4}$ Transducer Science and Technology, $\mathrm{MESA}^{+}$, University of Twente, the Netherlands

\section{bram.krijnen@demcon.nl}

\begin{abstract}
Position sensing in MEMS is often based on the principle of varying capacitance [1]. Alternative position sensing principles include using integrated optical waveguides [2] or varying thermal conductance [3]. Lantz et al demonstrated a thermal displacement sensor achieving nanometre resolution on a $100 \mu \mathrm{m}$ range. However a multi-mask production process and manual assembly were needed to fabricate this displacement sensor. In this work we present a 1-DOF thermal displacement sensor integrated with an actuated stage, and its experimental characterization. The system was fabricated in the device layer of a silicon-on-insulator (SOI) wafer using a single-mask process.
\end{abstract}

The thermal displacement sensor consists of two U-shaped resistive heaters in a differential configuration as shown in figure 1. Its temperature distribution depends on the stage position, because the amount of overlap with the stage affects the cooling efficiency to the stage dominated by thermal conductance through air. The temperature variation is measured by exploiting the temperature-dependence of the electrical resistivity of silicon: Applying the same voltage on both heaters, the stage displacement is measured by the difference between the heater currents.

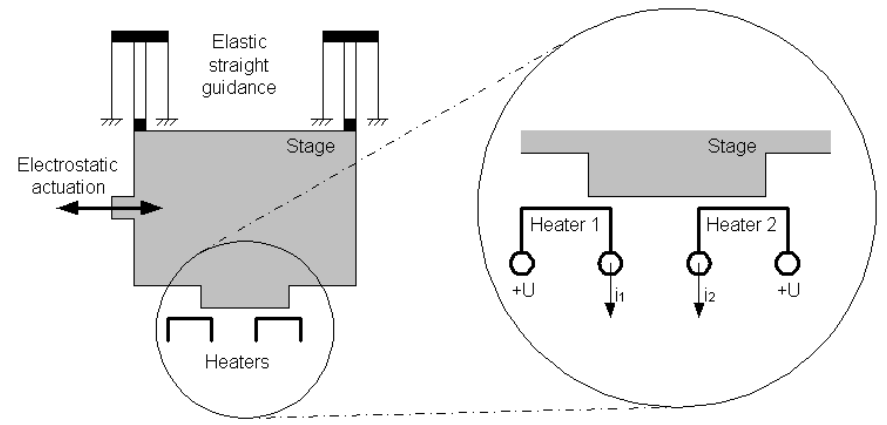

Figure 1: Schematic design of the integrated thermal displacement sensor. 


\section{Design and fabrication}

Figure 2 shows the fabricated sensor at maximum stage displacement. The legs of the U-shaped heaters and the sensing part have a length of $100 \mu \mathrm{m}$ and $60 \mu \mathrm{m}$ respectively. Their width measures $3 \mu \mathrm{m}$ and the height $25 \mu \mathrm{m}$. The air-gap between the heaters and the stage is $3 \mu \mathrm{m}$ wide. The sensor was bulk-micromachined in a highly boron-doped $\left(\approx 5^{*} 10^{-18} \mathrm{~cm}^{-3}\right) 25 \mu \mathrm{m}$ thick device layer of a SOI wafer. After deep reactive-ion etching (DRIE), the structures were released from the substrate by etching the buried oxide in HF-vapour.

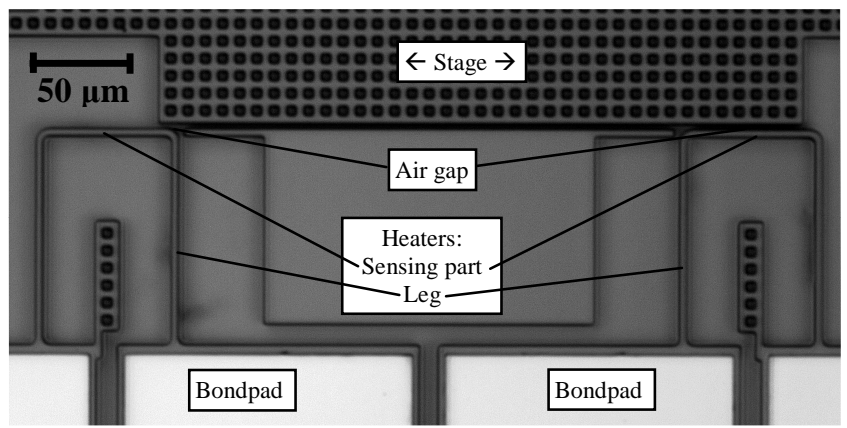

Figure 2: Microscope image of the sensor with the stage in its rightmost position.

The thermo-electrical equilibrium for maximum stage displacement was modelled in COMSOL and shown in figure 3. Applying a constant heater voltage of $9 \mathrm{~V}$, the maximum heater temperature for minimum stage overlap is $876 \mathrm{~K}$ versus $803 \mathrm{~K}$ at maximum overlap. The contribution of heat-transfer towards the stage at full overlap is about $10 \%$ of the total dissipated power, where remaining heat is lost directly towards the substrate and through the legs.

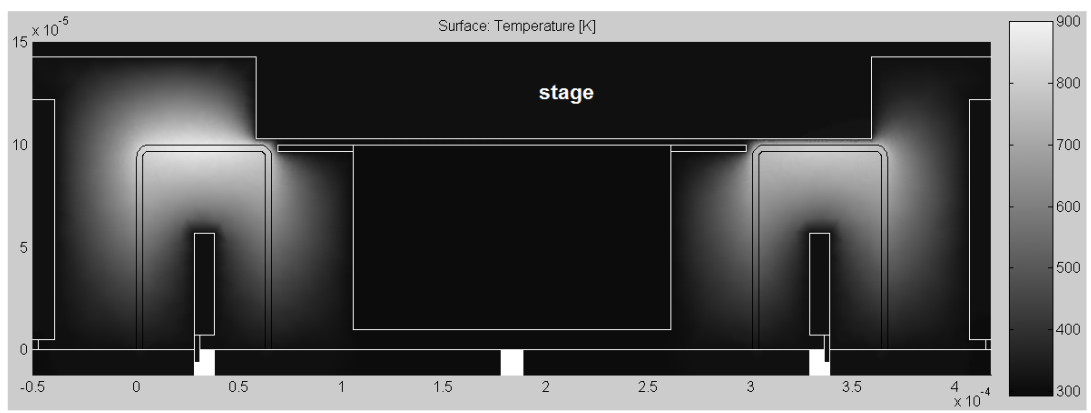

Figure 3: FEM temperature profile of the sensor with the stage in rightmost position. 


\section{Experimental results}

The sensor shown in figure 2 was experimentally characterized. The individual heater resistances vary by about $20 \Omega$ over the full displacement range, as shown in figure 4 . The resistance difference, caused by fabrication tolerances, was about $15 \Omega$ and contributes to an offset of the differential measurement.

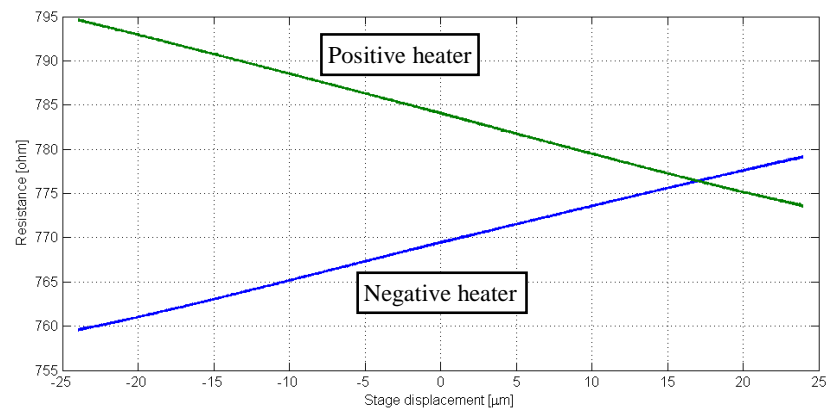

Figure 4: Measured resistance of both heaters as function of stage displacement.

The measurement signal after differential current-to-voltage amplification is shown in figure 5 (top). The mean sensitivity was $106.1 \mathrm{mV} / \mu \mathrm{m}$, which corresponds to a differential heater current of $12.6 \mu \mathrm{A} / \mu \mathrm{m}$. The 1 -sigma noise at $30 \mathrm{~Hz}$ bandwidth corresponds to less than $3 \mathrm{~nm}$. The total power consumption of the two heaters was about $209 \mathrm{~mW}$ and remained almost independent of the stage position. The thermal time constant of each heater is approximately $160 \mu \mathrm{s}$.
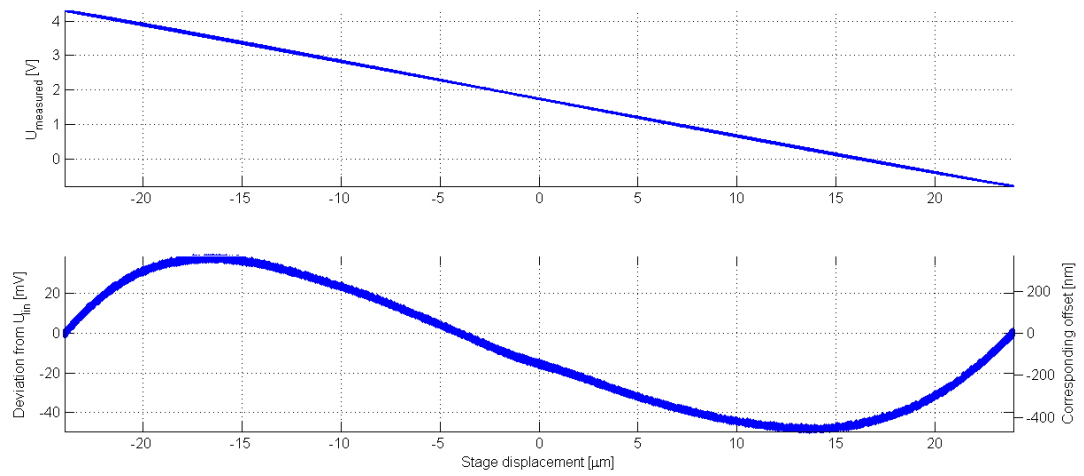

Figure 5: Measurement voltage as a function of the stage displacement (top) and nonlinearity of the sensor using the deviation from linear fit (bottom). 
The nonlinearity of the sensor signal, as shown in figure 5 (bottom), is primarily caused by the non-uniform temperature distribution of the heater: The sensor sensitivity, as shown in figure 6, is higher in the middle region due to locally higher heater temperatures.

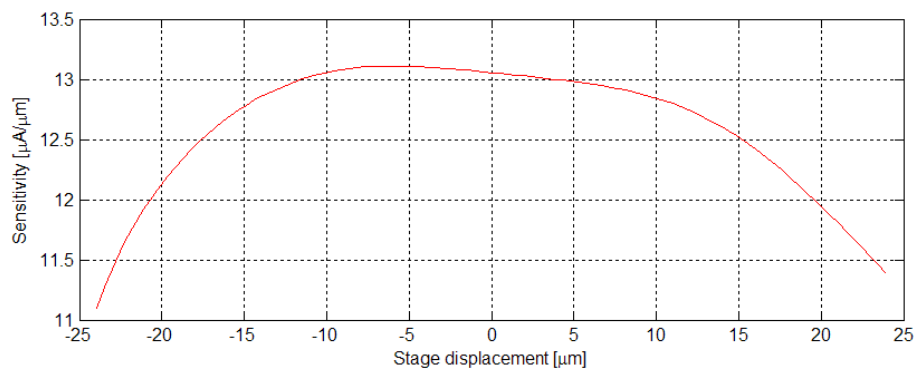

Figure 6: Derived sensitivity as function of stage displacement.

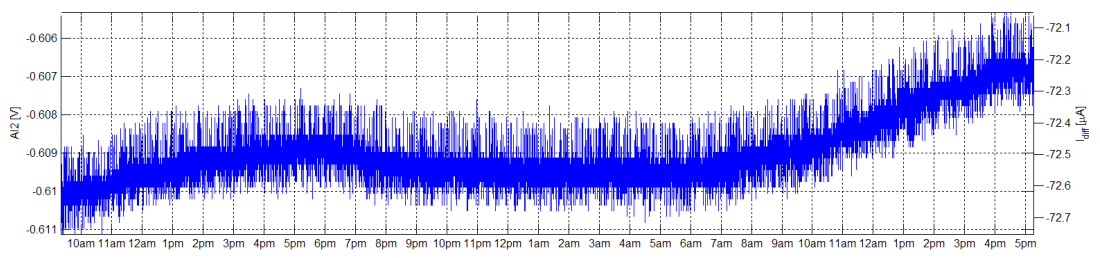

Figure 7: Drift of the differential signal in the neutral position during 32 hours.

Figure 7 shows the sensor drift with the stage in the neutral position under normal laboratory conditions after 1 week run-in. The drift of the single heater current over a period of 32 hours was $27 \mu \mathrm{A}$, while the differentially compensated drift was only $0.4 \mu \mathrm{A}(32 \mathrm{~nm})$. The drift might be further reduced by addition of reference sensors.

\section{References:}

[1] A.A. Kuijpers, Micromachined capacitive long-range displacement sensor for nano-positioning of micro-actuator systems, $\mathrm{PhD}$ thesis, University of Twente, Enschede, the Netherlands (2004)

[2] Y. Bellouard, et al., High accuracy micro-displacement sensor with integrated optics-based detection means, IEEE 2005 International Conference on Robotics and Automation, ICRA 2005

[3] M.A. Lantz, et al., A micromechanical thermal displacement sensor with nanometre resolution, Nanotechnology 16 p1089-1094 (2005) 